\title{
Elastic Morphing of 2D and 3D Objects on a Shape Manifold
}

\author{
C. Samir ${ }^{1}$, P. Van Dooren ${ }^{1}$, D. Laurent ${ }^{1}$, K.A. Gallivan ${ }^{2}$, and P.-A. Absil ${ }^{1}$ \\ 1 Dept of Mathematical Engineering, Université catholique de Louvain, Belgium \\ 2 Dept of Mathematics, Florida State University, Tallahassee, FL 32306, USA
}

\begin{abstract}
We present a new method for morphing 2D and 3D objects. In particular we focus on the problem of smooth interpolation on a shape manifold. The proposed method takes advantage of two recent works on $2 \mathrm{D}$ and $3 \mathrm{D}$ shape analysis to compute elastic geodesics between any two arbitrary shapes and interpolations on a Riemannian manifold. Given a finite set of frames of the same (2D or $3 \mathrm{D})$ object from a video sequence, or different expressions of a 3D face, our goal is to interpolate between the given data in a manner that is smooth. Experimental results are presented to demonstrate the effectiveness of our method.
\end{abstract}

\section{Introduction}

There has been an increasing interest in recent years in analyzing shapes of 3D objects. Advances in shape estimation algorithms, 3D scanning technology, hardware-accelerated 3D graphics, and related tools are enabling access to highquality $3 \mathrm{D}$ data. As such technologies continue to improve, the need for automated methods for analyzing shapes of 3D objects will also grow. In terms of characterizing 3D objects, for detection, classification, morphing, and recognition, their shape is naturally an important feature. It already plays an important role in medical diagnostics, object designs, database search, and some forms of 3D face animation. Focusing on the last topic, our goal in this paper is to develop a new method for morphing $2 \mathrm{D}$ curves and $3 \mathrm{D}$ faces in a manner that is smooth and more "natural", i.e. interpolate the given shapes smoothly, and capture the optimal and elastic non-linear deformations when transforming one face to another.

\subsection{Previous Work}

During the last few years several application driven methods have been proposed for different purposes. For example, Crouch et al. [2] implemented the de Casteljau algorithm on Lie groups and on $m$-dimensional spheres under some boundary conditions. More recently, Jakubiak et al. [5] presented a geometric algorithm to generate splines of an arbitrary degree of smoothness on a Euclidean space and then extended it to matrix Lie groups. They applied their algorithm to design a smooth motion of a 3D object in space. Using an unrolling and unwarping procedure on a Riemannian manifolds, Kume et al. 9] developed a new method to fit

M. Kamel and A. Campilho (Eds.): ICIAR 2009, LNCS 5627, pp. 563 572 2009.

(C) Springer-Verlag Berlin Heidelberg 2009 
smooth curves through a series of landmark shapes. Using a discrete construction based on the de Casteljau algorithm, Altafini [1] proposed an interpolation method by smooth closed-form curves on the Lie group of rigid body motions. A new version of the de Casteljau algorithm, introduced by Popeil et al. 13. generalizes Bézier curves on a connected Riemannian manifold. They replaced line-segments in the classical de Casteljau algorithm by geodesics segments on the manifold. Their algorithm was implemented and tested on a data set in a two-dimensional hyperbolic space.

Most techniques for morphing, both 2D and 3D shapes, are based on a sparse set of user selected feature points. These are then used to establish the correspondences which in turn are used for interpolation. Much of the work done in morphing has been done on 2D metamorphosis. 3D morphs on the other hand change the geometry of the object and are then harder to compute and control. A good summary of previous work on the 3D morphing problem as that of Lazarus et al. [11. The authors note that there are unlimited ways to interpolate between different 3D objects. Hughes [4] proposed a method working in the Fourier domain. This provided novel ways to control the morph by treating frequency bands with different functions of time. Whitaker et al. [17] performed morphing through the application of evolution equations.

\subsection{Our Approach}

Given a finite set of points on a shape manifold, we want to fit the given data with a smooth and continuous curve. One efficient way to reach this goal, is to apply the de Casteljau algorithm [5] to interpolate between the given data. The goal here is to use classical polynomial interpolations, such as Bézier or Lagrange. Introduced a few decades ago, those interpolations has been defined and applied to the Euclidean plane. Recently, the de Casteljau algorithm served as a tool to generalize them on any Riemannian manifold, given a way to compute geodesics on the manifold [12].

Based on recent work on 2D and 3D shape analysis, we will first introduce an efficient method to compute geodesics on a shape manifold between any two arbitrary closed curves in $\mathbb{R}^{n}$. We will then generalize it to surfaces of genus zero. To this end, we will choose a representation for curves and surfaces in order to facilitate their analysis as elements of infinite non-linear manifolds 14. Other methods to compute geodesics on a shape manifold could be applied for the same purpose. But we will show that our choice is based on some advantages of this method: the smoothness of the resulting curve, the non-rigidity of the observed object, and the non-linearity of transformations going from one control point to another.

The rest of this paper is organized as follows. Detailed specific examples are given in Section 2. A Riemannian analysis of closed curves in $\mathbb{R}^{3}$ is presented in Section 3, with its extension to a Riemannian analysis of facial surfaces. The notion of smoothing (morphing of 3D faces) on a shape manifold is applied to curves and facial surfaces in Section 4 and the paper finishes with a brief summary in Section 5. 


\section{Some Specific Examples of Manifolds}

We will give two examples in order to make the reader familiar with the Lagrange and Bézier fitting curves obtained by the de Casteljau and Neville-Aitken algorithms 10 .

\subsection{On Euclidean Plane}

In this section we will not re-visit the literature on interpolation polynomials in detail, but we will give some examples on the Euclidean plane to help understanding the extension of this simple case to a Riemannian manifold. Bézier and Lagrange fitting curves for a given set of points in $\mathbb{R}^{2}$ are shown in figure 1(a). Observe that the Lagrange interpolation passes through the control points, while the Bézier curve starts at the first control point and ends at the last one without passing through the intermediate control points.



(a)

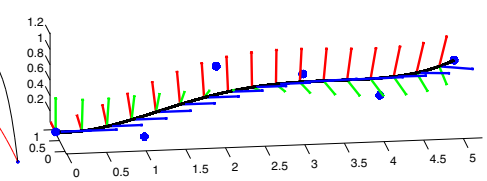

(b)

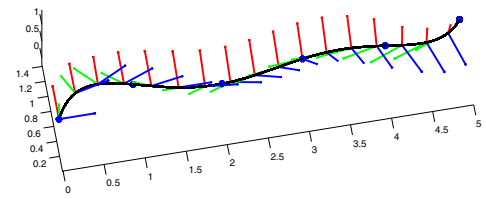

(c)

Fig. 1. (a): Bézier and Lagrange on Euclidean plane, (b): Bézier on $S O(3)$, and (c): Lagrange on $S O(3)$

\section{$2.2 \quad$ Interpolation on $S O(3) \times \mathbb{R}^{3}$}

In this section we will consider the problem of fitting curves to a finite set of control points derived from a continuous observation of a rigid transformation of a $3 \mathrm{D}$ object in space. A similar idea was applied in 5 , to build a trajectory of a satellite in space, where only rotations and translations were considered.

We are given a finite set of positions (3D coordinates) in $\mathbb{R}^{3}$, and finite set of rotations, at different instants of time. Our goal is to compute a rigid body motion the object will pass through (or close to) the given positions, and will rotate by the given rotations, at the given instants. The Bézier curve is obtained by the de Casteljau algorithm ( see details in [1]) and the Lagrange curve by the Neville-Aitken algorithm in $S E(3)$. As shown in figure 1, we obtain different fitting curves using Bézier in figure 1(b) and Lagrange in figure 1(c). In order to visualize the end effect, the two figures show the motion of a rigid body, where position is given by the curve in $\mathbb{R}^{3}$, and rotation is displayed by rotating axes. 


\section{Interpolation on Shape Manifold}

The crucial tool in applying Neville-Aitken's and de Casteljau's algorithms on a manifold is a method that constructs a geodesic between any pair of points. In this section, we consider methods for constructing geodesics between pairs of curves and between pairs of surfaces.

\subsection{Geodesic between Curves}

Here we adopt the approach presented in Joshi et al. 6] because it greatly simplifies the elastic shape analysis. The main steps are: (i) defining a space of closed curves of interest, (ii) imposing a Riemannian structure on it using the elastic metric, and (iii) computing geodesic paths under this metric. These geodesic paths can then be interpreted as optimal elastic deformations of curves.

For the interval $I \equiv[0,2 \pi]$, let $\beta: I \rightarrow \mathbb{R}^{3}$ be a parameterized curve with a non-vanishing derivative everywhere. We represent its shape by the function:

$$
q: I \rightarrow \mathbb{R}^{3}, q(s)=\frac{\dot{\beta}(s)}{\sqrt{\|\dot{\beta}(s)\|}} \in \mathbb{R}^{3} .
$$

Where, $\|\cdot\| \equiv \sqrt{(\cdot, \cdot)_{\mathbb{R}^{3}}}$, and $(\cdot, \cdot)_{\mathbb{R}^{3}}$ are taken to be the standard Euclidean inner product in $\mathbb{R}^{3}$. The quantity $\|q(s)\|$ is the square-root of the instantaneous speed of the curve $\beta$, whereas the ratio $\frac{q(s)}{\|q(s)\|}=\frac{\dot{\beta}(s)}{\|\dot{\beta}(s)\|}$ is the direction for each $s \in[0,2 \pi)$ along the curve. Let $\mathcal{Q}$ be the space of all square integrable functions in $\mathbb{R}^{3}$,

$$
\mathcal{Q} \equiv\left\{q=\left(q_{1}, q_{2}, q_{3}\right) \mid q(s): I \rightarrow \mathbb{R}^{3}, q(s) \neq 0, \forall s\right\} .
$$

The closure condition for a curve $\beta$ requires that $\int_{I} \dot{\beta}(s) d s=0$, which translates to $\int_{I}\|q(s)\| q(s) d s=0$. We define a mapping $\mathcal{G}: \mathcal{Q} \mapsto \mathbb{R}^{4}$, with components:

$\mathcal{G}_{1}=\int_{I} q_{1}(s)\|q(s)\| d s, \mathcal{G}_{2}=\int_{I} q_{2}(s)\|q(s)\| d s, \mathcal{G}_{3}=\int_{I} q_{3}(s)\|q(s)\| d s, \mathcal{G}_{4}=\int_{I}\|q(s)\|^{2} d s=1$.

The space obtained by the inverse image $\mathcal{C}=\mathcal{G}^{-1}(\mathbf{0})$ is the space of all closed curves of unit length, and this representation is invariant under translation and scaling. $\mathcal{C}$ is endowed with a Riemannian structure using the following metric: for any two tangent vectors $v_{1}, v_{2} \in T_{q}(\mathcal{C})$, we define

$$
\left\langle v_{1}, v_{2}\right\rangle=\int_{I}\left(v_{1}(s), v_{2}(s)\right)_{\mathbb{R}^{3}} d s .
$$

Next, we want a tool to compute geodesic paths between arbitrary elements of $\mathcal{C}$. There have been two prominent numerical approaches for computing geodesic paths on nonlinear manifolds. One approach uses the shooting method [8] where, given a pair of shapes, one finds a tangent direction at the first shape such that its image under the exponential map gets as close to the second shape as possible. 
We will use another, more stable approach that uses path-straightening flows to find a geodesic between two shapes. In this approach, the given pair of shapes is connected by an initial arbitrary path that is iteratively "straightened" so as to minimize its length. The path-straightening method, proposed by Klassen et al 7, overcomes some of the practical limitations in the shooting method. Other authors, including Schmidt et al. [15] and Glaunes et al [3, have also presented other variational techniques for finding optimal matches. Given two curves, represented by $q_{0}$ and $q_{1}$, our goal is to find a geodesic between them in $\mathcal{C}$. Let $\alpha:[0,1] \rightarrow \mathcal{C}$ be any path connecting $q_{0}, q_{1}$ in $\mathcal{C}$, i.e. $\alpha(0)=q_{0}$ and $\alpha(1)=q_{1}$. Then, the critical points of the energy

$$
E[\alpha]=\frac{1}{2} \int_{0}^{1}\langle\dot{\alpha}(t), \dot{\alpha}(t)\rangle d t,
$$

with the inner product defined in Eqn. 1, are geodesics in $\mathcal{C}$ (this result is true on a general manifold [16]). As described by Klassen et al. 7] (for general shape manifolds), one can use a gradient approach to find a critical point of $E$ and converge to a geodesic. The distance between the two curves $q_{0}$ and $q_{0}$ is given by the length of the geodesic $\alpha$ :

$$
d_{c}\left(q_{1}, q_{2}\right)=\int_{0}^{1}(\langle\dot{\alpha}(t), \dot{\alpha}(t)\rangle)^{1 / 2} d t .
$$

We call this the elastic distance in deforming the curve represented by $q_{0}$ to the curve represented by $q_{1}$.

We will illustrate these ideas using some examples. Some examples of elastic matching between planar shapes are presented in Figure 2, Nonlinearity of matching between points across the two shapes emphasizes the elastic nature of this matching. One can also view these paths as optimal elastic deformations of one curve to another.
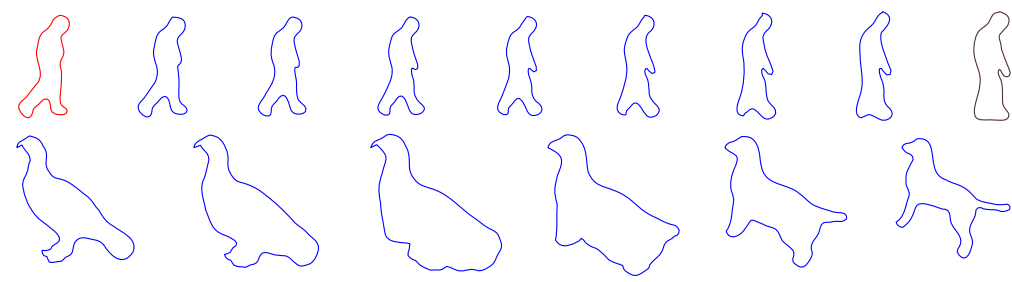

Fig. 2. Elastic deformation (geodesic) between 2D curves from Kimia database

\subsection{Geodesic between Faces}

Analyzing the morphing of a surface is much more complicated due to the corresponding difficulty in analyzing shapes of surfaces. The space of parameterizations of a surface is much larger than that of a curve, and this hinders an analysis of deformation in a way that is invariant to parameterization. One solution is to 


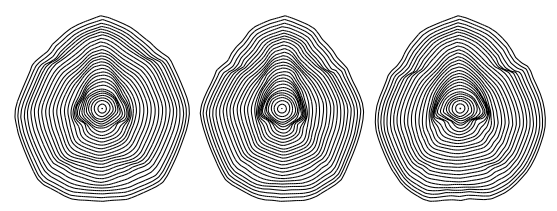

Fig. 3. Representation of facial surfaces as indexed collection of closed curves in $\mathbb{R}^{3}$

restrict to a family of parameterizations and perform shape analysis over that space. Although this can not be done for all surfaces, it is natural for certain surfaces such as the facial surfaces as described next.

Using the approach of Samir et al. [14, we can represent a facial surface $S$ as an indexed collection of facial curves, as shown in Figure 3. Each facial curve, denoted by $c_{\lambda}$, is obtained as a level set of the (surface) distance function from the tip of the nose; it is a closed curve in $\mathbb{R}^{3}$. As earlier, let $d_{c}$ denote the geodesic distance between closed curves in $\mathbb{R}^{3}$, when computed on the shape space $\mathcal{S}=\mathcal{C} /(S O(3) \times \Gamma)$, where $\mathcal{C}$ is the same as defined in the previous section except this time it is for curves in $\mathbb{R}^{3}$, and $\Gamma$ is the set of all parameterizations. A surface $S$ is represented as a collection $\cup_{\lambda} c_{\lambda}$ and the elastic distance between any two facial surfaces is given by: $d_{s}\left(S_{1}, S_{2}\right)=\sum_{\lambda} d_{c}(\lambda)$, where

$$
d_{c}(\lambda)=\inf _{O \in S O(3), \gamma \in \Gamma} d_{c}\left(q_{\lambda}^{1}, \sqrt{\dot{\gamma}} O q_{\lambda}^{2}(\gamma)\right) .
$$

Here $q_{\lambda}^{1}$ and $q_{\lambda}^{2}$ are $q$ representations of the curves $c_{\lambda}^{1}$ and $c_{\lambda}^{2}$, respectively. According to this equation, for each pair of curves in $S_{1}$ and $S_{2}, c_{\lambda}^{1}$ and $c_{\lambda}^{2}$, we obtain an optimal rotation and re-parameterization of the second curve. To put together geodesic paths between full facial surfaces, we need a single rotational alignment between them, not individually for each curve as we have now. Thus we compute an average rotation:

$$
\hat{O}=\operatorname{average}\left\{O_{\lambda}\right\},
$$

using a standard approach, and apply $\hat{O}$ to $S_{2}$ to align it with $S_{1}$. This global rotation, along with optimal re-parameterizations for each $\lambda$, provides an optimal alignment between individual facial curves and results in shortest geodesic paths between them. Combining these geodesic paths, for all $\lambda$ 's, one obtains geodesic paths between the original facial surfaces.
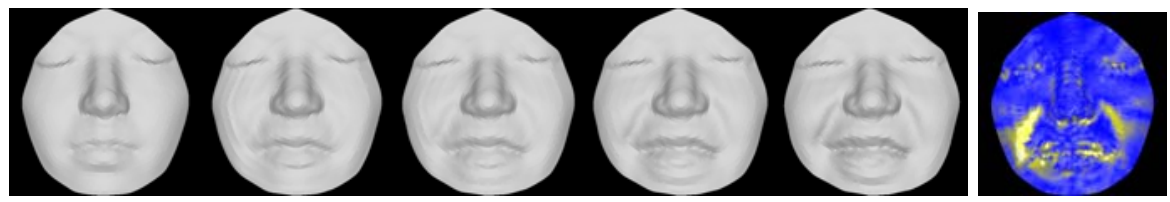

Fig. 4. Geodesic path between two facial surfaces, and the corresponding magnitude of deformation 


\section{Experimental Results}

In this section, we present some examples and discuss the effectiveness of our method. At each step of the interpolation, optimal deformations between two shapes are computed using geodesics on a shape manifold, as segments were used in the classical de Casteljau algorithm on the Euclidean plane. Note that all shapes are extracted from real data and are generated fully automatically without any user interaction.

\subsection{Examples Derived from Video-Sequences}

In the first example (see figure 5), curves are extracted from video sequences of growing leaves. In the second example (see figure [6), curves are extracted from a video sequence of a human walk. In each example, only four key frames are selected to be used as control points for interpolation. Curves are then extracted and represented as a vector of 100 points. Recall that shapes are invariant under rotation, translation, and re-parameterization. Thus, the alignment between the given curves is implicit in geodesics which makes the morphing process fully automatic. In figures 5 and [6, the first three rows show optimal deformations between ending shapes and the morphing sequences are shown in the last two rows. Thus, the fourth row shows Lagrange interpolation, and the last row shows Bézier fitting curve. It is clear from figures 5 and 6 that Lagrange interpolation gives (visually) a good morphing and passes through the given data.

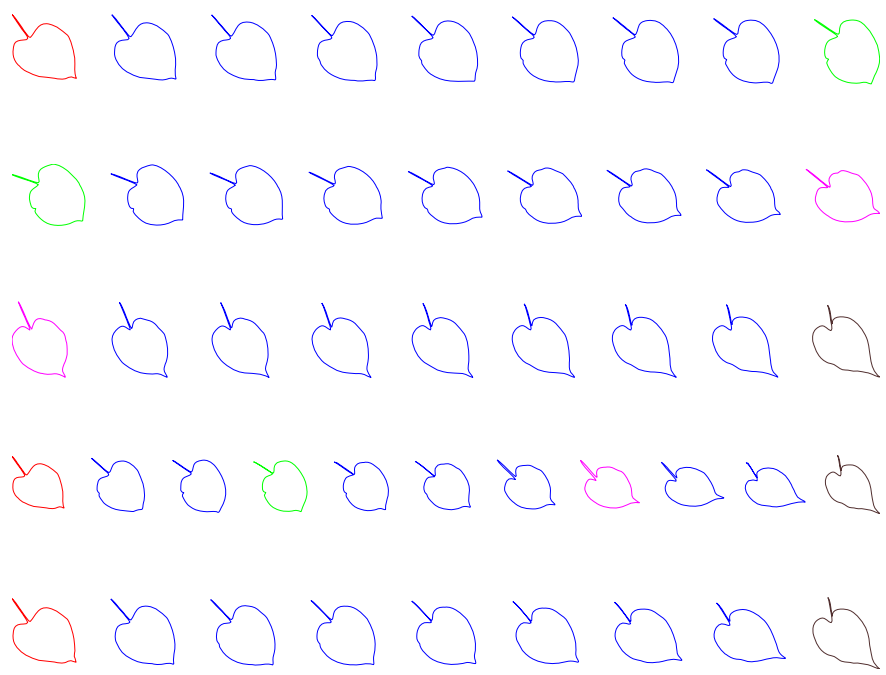

Fig. 5. First three rows: geodesic between the ending shapes (leaf contours). Fourth row: Lagrange interpolation between four control points (ending points in previous rows). Last row: Bézier fitting curve using the same control points. 
$\int$

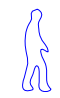<smiles>C1CCCC1</smiles><smiles>[C]1CCCC1</smiles><smiles>C1CCCCC1</smiles><smiles>[C]1CCCC1</smiles><smiles>[C]1CCCC1</smiles><smiles>C1CCCC1</smiles><smiles></smiles><smiles>C1CCC2(CC1)CCCC2</smiles><smiles>C1=CCCCC1</smiles><smiles>C1=CCCCC1</smiles><smiles>C1=CCCCC1</smiles><smiles>C1=CCCCC1</smiles><smiles>C1=CC2CCCCC12</smiles><smiles>C1=CCCCC1</smiles><smiles>C1=CCCCC1</smiles><smiles>C1CCCCC1</smiles><smiles>C1=CC(C2CCCC2)CCC1</smiles><smiles>c1ccccc1</smiles><smiles>c1ccccc1</smiles><smiles>c1ccccc1</smiles><smiles>c1ccccc1</smiles><smiles>C=CC=CC=C</smiles><smiles>c1ccccc1</smiles><smiles></smiles><smiles>[CH]1C=CC=C1[C]1C=C1</smiles><smiles>C1=CC=C1</smiles><smiles></smiles><smiles>C1=CC2C=CC(C=C2)C1</smiles><smiles>C1=CC=C1</smiles><smiles>c1ccccc1</smiles>

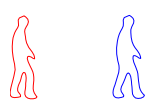<smiles>[C]1CCC1</smiles><smiles>C1CCCCC1</smiles><smiles>C1=CCCCC1</smiles><smiles>C1=CCCCC1</smiles><smiles>c1ccccc1</smiles><smiles>c1ccccc1</smiles><smiles>c1ccccc1</smiles>

Fig. 6. First three rows: geodesic between the ending shapes (human silhouettes from gait). Fourth row: Lagrange interpolation between four control points (ending points in previous rows). Last row: Bézier fitting curve using the same control points.
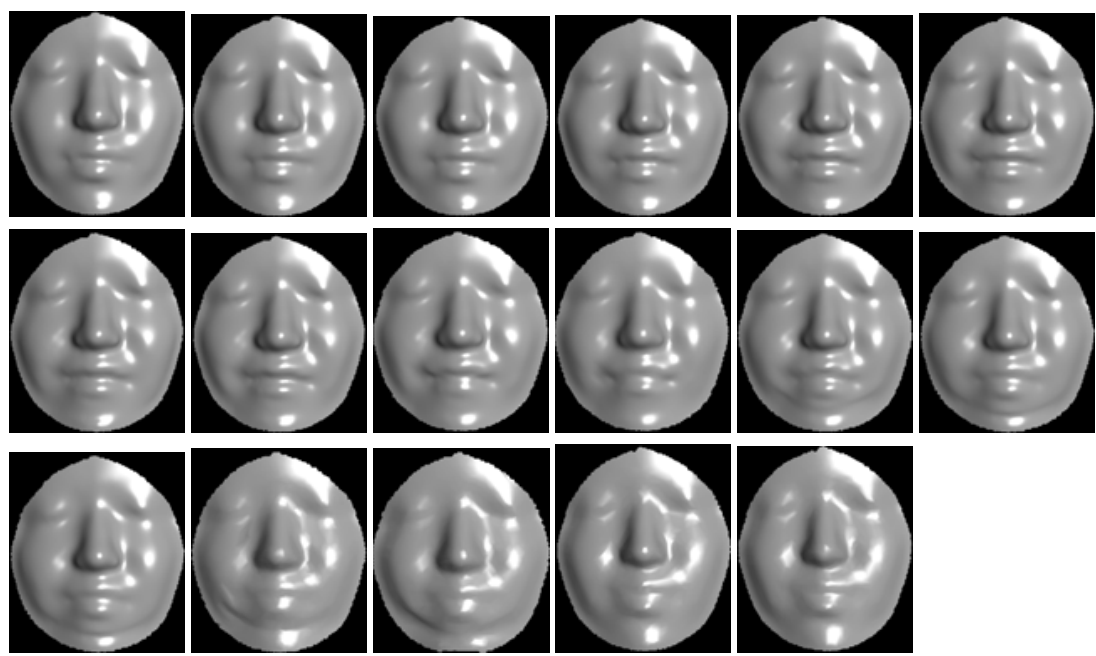

Fig. 7. Morphing 3D faces by applying Lagrange interpolation on four different facial expressions of the same person (1:4:17)

\subsection{D Face Morphing}

In this example we show how to build an animation of $3 \mathrm{D}$ faces using different facial surfaces that represent the same person under different facial expressions. 
In contrast with some previous methods that show morphing between faces as a deformation from one face to another, which could be obtained here by a geodesic between two faces, our goal is to provide a way to build a morphing that includes a finite set of faces. So, as shown in figure 7, we can use different facial expressions (four in the figure) and make the animation start from one face and pass through different facial expressions using Lagrange interpolation on a shape manifold. As mentioned above, no manual alignment is needed. Thus, the animation is fully automatic. In this experiment, we represent a face as a collection of 17 curves, and each curve is represented as a vector of 100 points. The method proposed in this paper can be applied to more general surfaces if there is a natural way of representing them as indexed collections of closed curves. For more details, we refer the reader to 14 .

\section{Summary}

We have introduced a new method for 2D and 3D shape metamorphosis based on Neville-Aitken's and de Casteljau's algorithms on a shape manifold. A fully automatic method to morph a shape passing through (or close to) finite set of other shapes. We have shown some examples using 2D curves from a walkobservation, leaf-growth, and 3D faces to demonstrate the effectiveness of our method. The method is applicable to other object parameterizations. All that is required is a method to compute a geodesic between pairs of objects.

\section{Acknowledgment}

This paper presents research results of the Belgian Network DYSCO (Dynamical Systems, Control, and Optimization), funded by the Interuniversity Attraction Poles Programme, initiated by the Belgian State, Science Policy Office. The scientific responsibility rests with its authors.

\section{References}

1. Altafini, C.: The de casteljau algorithm on se(3). In: Nonlinear control in the Year 2000, pp. 23-34 (2000)

2. Crouch, P., Kun, G., Leite, F.S.: The de casteljau algorithm on the lie group and spheres. Journal of Dynamical and Control Systems 5, 397-429 (1999)

3. Glaunes, J., Qiu, A., Miller, M., Younes, L.: Large deformation diffeomorphic metric curve mapping. International Journal of Computer Vision 80, 317-336 (2008)

4. Hughes, J.F.: Scheduled fourier volume morphing. In: Computer Graphics (SIGGRAPH 1992), pp. 43-46 (1992)

5. Jakubiak, J., Leite, F.S., Rodrigues, R.C.: A two-step algorithm of smooth spline generation on Riemannian manifolds. Journal of Computational and Applied Mathematics, 177-191 (2006)

6. Joshi, S.H., Klassen, E., Srivastava, A., Jermyn, I.: A novel representation for riemannian analysis of elastic curves in $\mathrm{R}^{n}$, CVPR (2007) 
7. Klassen, E., Srivastava, A.: Geodesics between 3D closed curves using pathstraightening. In: Leonardis, A., Bischof, H., Pinz, A. (eds.) ECCV 2006. LNCS, vol. 3951, pp. 95-106. Springer, Heidelberg (2006)

8. Klassen, E., Srivastava, A., Mio, W., Joshi, S.: Analysis of planar shapes using geodesic paths on shape spaces. IEEE Patt. Analysis and Machine Intell. 26(3), 372-383 (2004)

9. Kume, A., Dryden, I.L., Le, H., Wood, A.T.A.: Fitting cubic splines to data in shape spaces of planar configurations. Proceedings in Statistics of Large Datasets, LASR, 119-122 (2002)

10. Lancaster, P., Salkauskas, K.: Curve and surface fitting. Academic Press, London (1986)

11. Lazarus, F., Verroust, A.: Three-dimensional metamorphosis: a survey. The Visual Computer, 373-389 (1998)

12. Lin, A., Walker, M.: Cagd techniques for differentiable manifolds, Tech. report, York University (July 2001)

13. Popeil, T., Noakes, L.: Bézier curves and $c^{2}$ interpolation in Riemannian manifolds. Journal of Approximation Theory, 111-127 (2007)

14. Samir, C., Srivastava, A., Daoudi, M., Klassen, E.: An intrinsic framework for analysis of facial surfaces. International Journal of Computer Vision 82, 80-95 (2009)

15. Schmidt, F.R., Clausen, M., Cremers, D.: Shape matching by variational computation of geodesics on a manifold. In: Franke, K., Müller, K.-R., Nickolay, B., Schäfer, R. (eds.) DAGM 2006. LNCS, vol. 4174, pp. 142-151. Springer, Heidelberg (2006)

16. Spivak, M.: A comprehensive introduction to differential geometry, vol. i \& ii. Publish or Perish, Inc., Berkeley (1979)

17. Whitaker, R., Breen, D.: Level-set models for the deformation of solid object. In: Third International Workshop on Implicit Surfaces, pp. 19-35 (1998) 